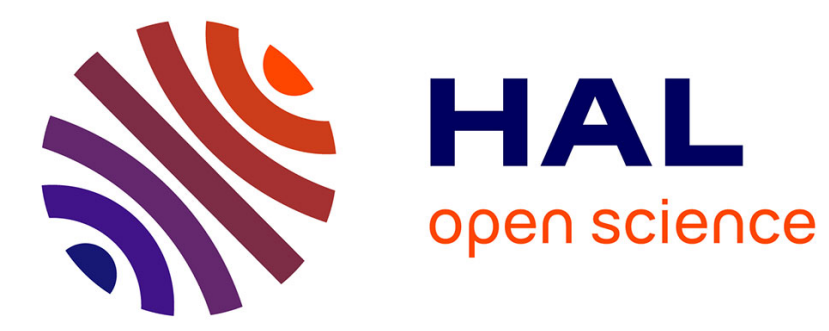

\title{
Quand l'objection de croissance révèle certains des impensés de la gauche
}

\author{
Fabrice Flipo
}

\section{To cite this version:}

Fabrice Flipo. Quand l'objection de croissance révèle certains des impensés de la gauche. Revue du MAUSS, 2009, 34, pp.265-279. 10.3917/rdm.034.0265 . hal-00958035

\section{HAL Id: hal-00958035 \\ https://hal.science/hal-00958035}

Submitted on 11 Mar 2014

HAL is a multi-disciplinary open access archive for the deposit and dissemination of scientific research documents, whether they are published or not. The documents may come from teaching and research institutions in France or abroad, or from public or private research centers.
L'archive ouverte pluridisciplinaire HAL, est destinée au dépôt et à la diffusion de documents scientifiques de niveau recherche, publiés ou non, émanant des établissements d'enseignement et de recherche français ou étrangers, des laboratoires publics ou privés. 


\section{Quand l'objection de croissance révèle certains des impensés de la gauche}

Par Fabrice Flipo

Résumé : cet article tente de montrer que l'opposition déclarée de Jean-Marie Harribey à la décroissance tient principalement à une lecture partielle et partiale des textes des objecteurs de croissance. Cette partialité n'agit pas au hasard et décrit plusieurs récurrences : la naturalisation de l'économie comme art du comportement " économe " (gain de temps, de productivité) et un déni du poids ontologique du symbolique dans l'organisation des sociétés humaines au profit d'un discours qui se concentre sur les moyens et non sur les fins.

Summary: this article claims that Jean-Marie Harribey's opposition to degrowth is mainly caused y a partial undestanding of its positions. This partiality isn't erratic, it is implicitly built on three claims : the naturalisation of economy understood as the art of the minimum effort (time sparing, productivity increasing) and a denial of the ontologic weight of symbols in human societies organisations which paves the way to a discours on means rather than on ends.

Jean-Marie Harribey, économiste marxiste, maître de conférences à l'université de Bordeaux, est l'auteur de multiples articles sur la décroissance ${ }^{1}$. On pourrait se réjouir du débat ainsi lancé. Pourtant il n'en est rien, car les objecteurs de croissance ne se reconnaissent pas dans ce que dit Jean-Marie Harribey. II n'y a pas de débat mais une gigantesque incompréhension. A quoi cela tient-il ? Nous essayons ici de dénouer les fils.

II serait long et fastidieux d'entreprendre un commentaire de texte systématique des articles de Jean-Marie Harribey sur la décroissance. Nous avons choisi ici de décortiquer l'un des plus récents, celui qui est destiné à l'Encyclopédie Universalis, que l'on peut trouver sur le site de son auteur à l'adresse suivante http://harribey.u-bordeaux4.fr/travaux/soutenabilite/decroissance-eu.pdf Le but est double : d'une part expliquer pourquoi, textes en main, le débat n'a pas lieu, et d'autre part proposer des explications au sujet des "points aveugles » qui sont sous-jacents aux incompréhensions. Avec l'espoir de permettre aux lecteurs de se faire une opinion plus consistante du sujet.

\section{Les filiations}

La première partie du texte de J.-M. Harribey entend retracer les filiations de la décroissance. Pour résumer en quelques mots, la décroissance est issue des conceptions pessimistes de l'économie, que l'auteur ramène à Malthus, de l'écologie politique, ramenée à Hans Jonas, Arne Naess et Ivan Illich, dépeints comme adversaires de la Raison, et de l'entropie, ramenée à Nicholas GeorgescuRoegen.

Ces filiations passent sous silence des points importants. Pas un mot sur les alternatives qui sont proposées par les différents courants de la décroissance: la consommation différente, la relocalisation, " plus de liens », la joie de vivre, le revenu maximum autorisé etc. Rien n'est dit des mouvements, ni de ce qu'ils font ni de ce qu'ils proposent, rien n'est dit sur la critique de la société de spectacle etc. De plus les trois filiations proposées sont tronquées :

- si les limites à la croissance sont pointées par les objecteurs de croissance, ce n'est pas pour souligner l'excès de pauvres et l'impossibilité de la terre à subvenir à leurs besoins, comme le faisait Malthus, mais pour montrer que la croissance est un phénomène qui n'est pas " gagnant-gagnant » comme l'avait théorisé Ricardo. La croissance a un coût caché, un « tiers exclu » : l'environnement, le Tiers-monde etc. II faut beaucoup de mise

1 Du côté de la décroissance : questions encore non résolues, Décroissance ou Neuvième Symphonie, Cahiers marxistes, octobre-novembre 2008; Que faire croître et décroître ?, Contretemps, février 2007; Les théories de la décroissance : enjeux et limites, Cahiers français, mars-avril 2007 ; Toute critique radicale est-elle recyclable dans la décroissance? Commentaires critiques sur deux ouvrages récents (Paul Ariés et Jean-Paul Besset), Contretemps, février 2007 ; Les dangers du discours sur la décroissance (avec Cyril Di Méo), 2006 etc. 
en scène pour ne pas le voir, d'où le souci de " protéger l'environnement mental " comme le dit Casseurs de Pub. Quand les yeux se sont enfin ouverts, il appert que poursuivre la croissance détruit les sociétés, pour différentes raisons. Changer l'économie pour que cette contrepartie disparaisse implique non pas seulement de " changer le contenu de la croissance " (du PIB) mais la décroissance (du PIB toujours). Un tel arrêt ne traduirait que la disparition des désirs futiles engendrés par la publicité et l'industrie culturelle, comme l'avait montré l'Ecole de Francfort - c'est tout le combat d'un journal comme Casseurs de $P u b$, dirigé par les mêmes personnes que le journal La Décroissance : Vincent Cheynet et Bruno Clémentin. Paul Ariès et Vincent Cheynet sont très explicites sur la question des inégalités ${ }^{2}$. On est très loin de l'argument de Malthus, et très loin de Malthus lui-même. Le débat ne s'inscrit pas dans les mêmes termes que celui qui avait opposé Marx à Malthus, un siècle et demi en arrière ; l'y ramener est extrêmement réducteur, ne rend pas justice aux arguments proposés, et finalement ne situe pas le courant de pensée dans les filiations dont il se réclame.

- l'objection vaut aussi pour la question de l'écologie. Dire que la décroissance serait proche de la deep ecology, telle qu'elle est connue en France, c'est accréditer l'idée que l'objection de croissance prendrait ses racines dans l'écologie réactionnaire, celle qui voit dans "l'humanité ", pris comme un tout indistinct, l'ennemi de "la planète ". Pourtant tel n'est pas le cas. Tout d'abord la deep ecology n'est pas la caricature popularisée en France par Luc Ferry. Arne Naess, fondateur de la deep ecology, dont le premier livre en français vient d'être publié ${ }^{3}$, était libertaire et pacifiste - impossible d'en faire un adepte du génocide! Comment peut-on se laisser ainsi dominer par les clichés de Luc Ferry ? Ensuite l'écologie à laquelle la décroissance se réfère n'est pas l'écologie new age, Paul Ariès a publié des livres sur ce sujet ${ }^{4}$ et chacun pourra constater en le lisant que le journal "Silence " est attaché à ne pas être confondu avec ces courants, qu'il ne faut pas confondre avec le souci d'une ouverture spirituelle à l'image d'un Pierre Rabhi par exemple. Le choix de Lovelock comme référence idéologique, tendant à accréditer l'idée d'une " nature-mère » est tout aussi contestable car l'illustre savant anglais cherche avant tout à nous expliquer que Gaïa est autorégulée et se relèverait très bien d'une disparition de l'humanité. Au contraire les objecteurs de croissance se distinguent de certains courants écologistes en ce qu'ils posent explicitement la question du partage des ressources qui est consécutive à la protection des écosystèmes. De même si Ellul et Charbonneau contestent " la technique » et la raison instrumentale qui en est à l'origine, c'est en tant que celle-ci est, comme le disait Marx, " un rapport à la nature ", mais aussi un rapport social - et en l'occurrence un rapport social oppresseur, car issu de pouvoirs centralisés sur lesquels le citoyen n'a plus de prise. Que ces penseurs aient de plus vécu leur spiritualité n'est pas plus étonnant ou difficile à comprendre que ne l'est la présence dans les fondateurs d'Attac, dont Jean-Marie Harribey est coprésident, de Témoignage Chrétien ou de... Golias, les éditions chrétiennes qui éditent la plupart des livres de Paul Ariès. Ce n'est donc pas au paganisme et au new age qu'il faut se référer.

- il est important de comprendre aussi que l'objection de croissance prend ses racines dans les courants libertaires, ce qui explique une méfiance forte envers l'Etat - un thème qui n'apparait jamais dans les travaux de Jean-Marie Harribey. L'une des racines de la décroissance est donc la pensée libertaire telle que celle de Murray Bookchin. Si la " modernité » est critiquée, c'est dans le sens d'une " technicisation » du monde, il suffit de relire Ellul et Charbonneau pour le constater. Cette «technique » tant critiquée ne conduit pas à l'abolition de toute technique, ce qui serait un non-sens, mais à la promotion d'autres techniques, qui furent appelées " alternatives », " vernaculaires » etc. par les E.F. Schumacher I. Illich etc. qui peuplent le panthéon de l'écologie politique dont il s'agit ici. Renier cette "technique " c'est renier les promesses capitalistes mais aussi les promesses productivistes, c'est souligner qu'on aurait tort d'oublier que les expériences "socialistes " ont eu le même culte de la même technique salvatrice - un culte qui est

2 On se reportera utilement à P. Ariès, Décroissance ou barbarie, Golias, 2005 ; V. Cheynet, Le choc de la décroissance, Paris, Seuil, 2005 ; S. Latouche, Le pari de la décroissance, Paris, Fayard, 2006.

3 http://www.mouvements.info/Arno-Naess-et-l-ecologie-politique.html

4 P. Ariès, Anthroposophie : enquête sur un pouvoir occulte, Golias, 2001. 
encore à l'œuvre chez les partisans de la "durabilité faible », qui pensent que "la technique " va résoudre les problèmes écologiques et sociaux posés par les modes de vie des pays " développés ». Les filiations établies par Harribey induisent donc en erreur, ce n'est pas à Malthus qu'il faut se référer mais par exemple à Kropotkine, au marxisme hétérodoxe de Moishe Postone ${ }^{5}$, qui critique le travail comme source de valeur, à l'Ecole de Francfort ou encore aux Luddites, qui s'autorisaient à s'en prendre aux forces productives et pas seulement aux « rapports de production ».

- l'entropie : c'est là en effet le troisième élément que la décroissance évoque classiquement pour expliquer aux économistes ce dont il est question. Mais celui ou celle qui aborde le sujet doit aussi reconnaître que l'argument de l'entropie n'est utilisé par les " décroissants » que comme contre-argument face aux... économistes ! Ces économistes qui, oublieux des leçons des Physiocrates, confondent produit brut et produit net - ce dernier étant le solde obtenu entre les avances et le produit matériel obtenue après production. La référence à l'entropie a une fonction simple : dire en langage économique que ce qui est non renouvelable ne sera pas renouvelé, et donc que l'avenir ne peut être assuré que par ce qui est renouvelable. Ce point n'a l'air de rien et pourtant il ouvre sur des questions d'une profondeur abyssale. Tout d'abord il suffit de constater que notre quotidien repose sur les ressources non renouvelables pour imaginer l'ampleur des changements qui sont nécessaires pour mettre sur pied une société qui serait ancrée dans les ressources renouvelables. Ensuite les ressources renouvelables n'existent pas en quantité illimitée - le bois n'est renouvelable que si l'on en prend pas plus que ce qui se renouvelle effectivement. II y a donc d'emblée à la fois une question de répartition et une question de retenue dans la puissance consommatrice dont il est possible de disposer qu'on peut appeler une éthique. Ces deux conditions particulières font que l'usage du renouvelable se construit socialement de manière très différente des ressources épuisables et disponibles "à volonté ». II repose sur la res communis qui était une évidence populaire avant les enclosures, et peut donc avoir un air de " retour en arrière ". Sans être propriété étatique, elle n'est pas non plus propriété privée - là où Jean-Marie Harribey se contente généralement de faire référence au "public " sans autre précision. Ancrée dans le domaine moral et politique, voire juridique, cette discussion se situe endeçà de l'économie, capitaliste ou socialiste, puisque toutes dont ont, de fait, considéré la nature comme une res nullius. Avec l'objection de croissance c'est la question du partage, et non celle du génocide, qui est à l'ordre du jour. La « simplicité volontaire » est un monde entier d'expérimentations militantes qui visent à se soustraire à l'imaginaire préfabriqué produit par la publicité, qui entreprennent donc de déconstruire cet imaginaire, dans lequel le marxisme est largement tombé puisque sa critique à l'égard des bienfaits (progrès) produits par le capitalisme est faible voire inexistante - il ne s'agirait que de partager ces bienfaits, ce qui malheureusement est impossible, et donc utopique au sens marxien du terme, en partie à cause de l'entropie.

Avec les filiations proposées par Jean-Marie Harribey, un lecteur honnête aura tendance à associer décroissance avec Malthus, un authentique réac, avec la deep ecology, qu'il ne connait que par l'entremise de Luc Ferry et son "Nouvel ordre écologique », et l'entropie, qui dit finalement que nous existons « pour-la-mort », pour reprendre le vocabulaire de Martin Heidegger. Cette première partie, tout en n'étant pas totalement fausse (Malthus a bien parlé de limites à la croissance etc.), est donc suffisamment parcellaire pour avoir aiguillé le lecteur sur des fausses pistes. Le résultat des occultations est d'avoir ancré l'objection de croissance dans la réaction. Et cela alors que les objecteurs de croissance défendent de fait des propositions quasiment identiques à celles de Jean-Marie Harribey ${ }^{6}$ ! Ce point, à lui seul, ne devrait-il pas poser question ? Comment un dialogue pourrait-il s'engager sur de si mauvaises fondations ? C'est un peu comme si, pour débattre avec le marxisme, on commençait par le présenter comme antisémite (en ne retenant que les écrits de Marx sur les Juifs), antihumaniste (en soulignant ses écrits sur les droits de l'Homme et les Lumières - "Aucun des prétendus droits de l'homme ne s'étend au-delà de

5 M. Postone, Temps, travail et domination sociale, Paris, Editions de Minuit, 2009.

6 Politis, Débat avec Serge Latouche, René Passet », Propos recueillis par Patrick Piro, ... LATOUCHE S. [2003] 
I'homme égoïste, au-delà de l'homme comme membre de la société civile, savoir un individu replié sur lui-même, sur son intérêt privé et son caprice privé, l'individu séparé de la communauté. Bien loin que l'homme ait été considéré, dans ces droits-là, comme un être générique, c'est au contraire la vie générique elle-même, la société, qui apparaît comme un cadre extérieur aux individus, une entrave à leur indépendance originelle. Le seul lien qui les unisse, c'est la nécessité naturelle, le besoin et l'intérêt privé, la conservation de leur propriété et de leur personne égoïste ${ }^{7}$ ), totalitaire, vu sa théorie de la révolution jointe à une absence de théorie de l'Etat, ainsi que l'expérience stalinienne. Pourrait-on débattre avec «les marxistes" avec un tel préalable ? Pourrait-on débattre avec les anticapitalistes si on commençait par grouper ensemble anticapitalisme de droite - car il en existe un - et anticapitalisme de gauche ? On comprend que les personnes visées ne voient pas bien de quoi on leur parle.

\section{Les objections}

La seconde partie du texte est composée de trois paragraphes qui ramassent les critiques faites à " la décroissance » telle qu'elle a été définie dans la première partie du texte. Nous allons les analyser en partant de l'hypothèse que ces trois critiques sont plus intéressantes par ce qu'elles révèlent des problèmes théoriques dans lesquels l'anticapitalisme classique se débat, face à l'écologie, que de la faiblesse des arguments de "la décroissance », puisque, comme nous l'avons vu, la décroissance telle que Jean-Marie Harribey la dépeint n'est pas celle qui est réellement existante.

Un premier paragraphe s'intitule " de la décroissance au refus du développement et du progrès ». Le titre témoigne à lui seul de la confusion que fait Jean-Marie Harribey entre le progrès productiviste et le progrès évoqué par les objecteurs de croissance. Ici Jean-Marie Harribey reproche surtout aux objecteurs de croissance de confondre le développement avec sa version capitaliste, qui assimile croissance et développement (Rostow). Pourtant les écrits des objecteurs de croissance sont clairs : leur objet est le productivisme, pas le capitalisme. Les objecteurs de croissance viennent après les expériences socialistes et soviétiques. Pour croire que la critique anticapitaliste équivaut à une critique antiproductiviste, il faudrait quand même expliquer pourquoi toutes les expériences socialistes à ce jour ont suivi la même pente ; il faudrait expliquer pourquoi les mouvements "socialistes » ou " anticapitalistes " actuels ont tant de mal à penser un monde sans croissance ; bref il faudrait prendre le temps de lire les arguments qui poussent les objecteurs de croissance à ne pas se satisfaire de l'anticapitalisme. Marx n'a laissé que quelques lignes sur « la terre et le travailleur », il n'a nullement élaboré de théorie de l'exploitation de la nature, il n'a jamais pensé que "les subsistances" (les biens acquis par les travailleurs avec leur salaire) atteindraient un niveau tel que la planète serait menacée. De plus peut-on ignorer que Marx a donné, avec le Capital, non seulement une critique sociale mais aussi un " grand récit » ? Peut-on ignorer que le terme "développement» vient de l'embryologie ? Ignorer l'importance de l'organicisme dans la pensée socialiste ? La volonté saint-simonienne et communiste de supprimer le politique, de remplacer le débat et l'opinion par la science et l'administration des choses ? Les questions seront nombreuses, nous n'allons pas toutes les mentionner, d'autant qu'elles peuvent se ramener à une seule remarque d'ordre général : Jean-Marie Harribey n'aborde pas ces questions. II " lit » le mouvement de la décroissance au travers d'un prisme économiste qui ignore la question de l'Etat et se compose de deux possibilités: libéralisme ou antilibéralisme. Les objecteurs de croissance par contre s'intéressent aux relations humaines dans leur globalité, ainsi qu'à l'Etat. Ils questionnent bien croissance et développement ; tous distinguent une forme ou une autre de progrès qu'ils opposent à une autre forme, productiviste; tous prennent position sur la question de l'universel, d'une manière qui tranche avec le libéralisme comme avec le marxisme classique. Cela n'apparaît pas, il ne reste qu'une condamnation générale.

Le second paragraphe s'interroge : "une décroissance sans bornes ? ». A nouveau la question, présentée comme l'un des points aveugles de la mouvance décroissante, est étonnante, car tous les " décroissants " ont répondu à cette question, par la négative, et cela à de maintes reprises - il serait fastidieux de toutes les citer. Comment peut-on penser que l'objection de croissance se ramène à la revendication d'une décroissance "sans bornes »- jusqu'à ce que mort s'ensuive, en somme? Et si tel était le cas, devrait-on s'inquiéter, sur le plan politique, de voir quelques mouvements prôner la décroissance sans en préciser le terme alors que nous baignons dans une

7 La question juive, Pléiade, Philosophie, p. 368. 
inquiétude quant à la reprise de la croissance ? Jean-Marie Harribey semble tenir, dans le domaine de la défense du productivisme, la posture que les économistes libéraux avaient adopté à l'encontre de Marx et sa critique du capitalisme : "Celui donc qui dévoile ce qu'il en est dans la réalité de l'utilisation capitaliste de la machinerie est accusé de ne pas vouloir du tout qu'on l'emploie : c'est un ennemi du progrès social! $»^{8}$. L'objection de croissance ne soutient nulle part la thèse d'une décroissance illimitée, on sera bien en mal de trouver une seule citation en ce sens ; elle vient au contraire ouvrir la question d'une société de décroissance. Après plus d'un siècle et demi de société de croissance, on comprend que les questions posées soient radicalement nouvelles. Les enjeux pratiques et théoriques d'une telle question sont gigantesques. Savoir jusqu'où décroitre est l'objet d'un vif débat au sein des mouvements d'objection de croissance, dont l'objet principal est de se réapproprier la question des besoins - cette question étant aujourd'hui le lieu d'un individualisme marchand forcené, dominé par la publicité et le " grand récit » de l'Homme qui domine la nature - une forme de progrès qui occulte les " externalités » et autres effets négatifs. Les objecteurs de croissance cherchent à toucher le consommateur, à le faire s'interroger, en un lieu difficile d'accès car il touche au " progrès », en effet, mais tel qu'il est défini par le discours dominant, comme " progrès des forces productives ", et il est réputé privé, comme le clame la thèse de la souveraineté du consommateur. Pour autant aucun objecteur de croissance ne pense que la planification est la solution à tout, l'expérience soviétique est passée par là. Une part importante de la réflexion est donc consacrée à la place du marché et de l'initiative individuelle dans une économie qui ne serait plus productiviste. Jean-Marie Harribey n'a pas plus de position que Marx lui-même sur ce sujet, n'ayant pas produit de théorie de l'Etat. Au contraire les objecteurs de croissance puisent explicitement dans les idées libertaires et républicaines, ainsi Takis Fotopoulos qui est généreusement cité dans le dernier livre de Serge Latouche, Le pari de la décroissance.

Jean-Marie Harribey passe ce sujet sous silence, il préfère se concentrer sur un sujet qui de fait est périphérique dans littérature et les mouvements d'objection de croissance : la question de la population. II est vrai qu'après avoir ancré la décroissance chez Malthus, on ne peut que parler de population. Pourtant aucun objecteur de croissance ne s'est focalisé sur cette question, au contraire l'enjeu principal c'est de faire de la consommation et du partage une question publique. Latouche a surtout ironisé sur le sujet, disant que la réduction de la population sera inévitable si nous ne réduisions pas notre mode de vie - le pronom " nous " désignant la classe moyenne des pays "développés " et non les pauvres du monde entier! Pour Latouche, contrairement à Malthus, les "limites " ne conduisent pas à la nécessité d'éliminer les pauvres pour éviter la dégradation de la planète, ce n'est pas une nuance mais une option idéologique totalement différente. Arne Naess de son côté a en effet parlé de réduction de la population mais là encore le parallèle avec Mathus est fallacieux. Si Naess évoque cette question c'est pour prendre acte de ce que tout économiste sait depuis que l'économie existe : il y a bien un choix à faire entre nombre et niveau de vie, car plus on sera nombreux plus les niveaux de vie individuels devront être faibles pour respecter l'égalité et les écosystèmes. Les objecteurs de croissance entendent bien mettre ce sujet sur la table, là où les partisans de la croissance occultent les conséquences d'une population et d'une consommation qui vont toujours croissantes, en particulier en France. Sur la question de la population, que d'aucuns vont promptement lier au nazisme, rappelons Naess fut, pendant la seconde guerre mondiale, un résistant. Peu d'entre nous peuvent se prévaloir de tels faits. Cela devrait suffire à ne pas imputer des intentions de génocide à la première personne évoquant la question de la population.

Dans le même paragraphe, Jean-Marie Harribey dit que la critique du PIB devrait aller vers une réflexion sur l'utilité sociale. Et là on s'étonne, et le mot est faible, on s'étouffe plutôt, devant une telle affirmation, car c'est là le souci central des objecteurs de croissance ! Un souci que JeanMarie Harribey a totalement ignoré dans la présentation du mouvement, en première partie... Alors, lecture réductrice? Héritage marxiste, qui se refuse à renoncer aux " progrès » issus du capitalisme, préservant du même coup la neutralité des marxismes historiques vis-à-vis de la technique et de ses progrès? Déception de ne pas avoir inventé la décroissance avant les autres? Refus total de toute approche libertaire ou républicaine ? Souci de plaire auprès des milieux marxistes classiques ? Règlement de comptes personnel avec Serge Latouche, qui fut son directeur de thèse? Incompétence sur le plan scientifique et technique, qui ne permet pas de

8 Le capital Livre I traduction Lefebvre pp495-496 
comprendre les enjeux actuels ? Ce n'est pas clair. Dans la même veine, la question de la désocialisation, pourtant largement traitée par la décroissance derrière le slogan « moins de biens plus de liens ", fait aussi partie de ces sujets qui ont occultés en première partie et que Jean-Marie Harribey présente ensuite comme étant importants et trop ignorés des objecteurs de croissance!

Le dernier paragraphe s'attache à quelques considérations épistémologiques (" une épistémologie contestable ? ") très révélatrices. II démarre en effet par un constat qui témoigne d'un total désaccord avec les objecteurs de croissance : il y aurait d'un côté le travail au sens anthropologique et de l'autre le travail au sens capitaliste. Voilà une affirmation d'un marxisme des plus classiques, qui passe d'emblée à côté de la critique du travail façon Marshall Sahlins. Or c'est bien cette dernière qui est le cœur de la critique décroissante - et plus largement anti-utilitariste. Sur de si mauvaises bases bien sûr l'anticapitalisme de la décroissance peut être jugé «paradoxal » - alors que du point de vue de l'objection de croissance, c'est l'antiproductivisme déclaré de Jean-Marie Harribey qui est suspect, car jugé peu conséquent et ambigu : comment peut-on être "écologiste " et " antiproductiviste " et continuer de demander la croissance des services publics ou même simplement ne pas comprendre qu'une décroissance est souhaitable ?

Vient ensuite un passage qui tombe comme un couperet : "pour les théoriciens de la décroissance et du refus du développement, la coupable est finalement la Raison [...] [les objecteurs de croissance mettent] sur le même plan la science et la croyance ". Là encore, c'est une erreur de lecture: la raison tant critiquée est la raison instrumentale, économique et "fabricatrice », pour nous référer au vocabulaire de Hannah Arendt. Et cette raison ne fut pas, jusqu'ici, dépendante du capitalisme - cela parce que, pour reprendre les distinctions opérées par Marx, l'anticapitalisme s'est focalisé sur les rapports de production et non sur les forces productives, dont il a encouragé la croissance. Le type de raison qui est recherchée par les objecteurs de croissance est différente: écologique, démocratique etc. les orientations sont diverses au sein des mouvements. Pour convaincre, Jean-Marie Harribey devrait nous expliquer à quoi correspond, sur le plan de la rationalité individuelle et collective, la " durabilité forte " qu'il défend par ailleurs. L'écologie politique a répondu : c'est un rapport non-instrumental à la nature, c'est donc considérer la nature non seulement comme un moyen mais aussi comme une fin en soi. En défendant la durabilité forte, Jean-Marie Harribey devrait donc tomber sous le coup des condamnations qu'il adresse à la deep ecology...

Un passage sur le rapport entre foi et croyance, enfin, viendra clore le tout, et cherchera à nouveau à accréditer l'idée d'une unité foncière entre des courants qui sont loin de tout partager. Harribey affirme ceci : "sur quoi fonder l'écologie demande Goldsmith ? Pas sur la science mais sur la foi répond-il. Il s'agit donc de réenchanter le monde, ainsi que le dit aussi Latouche ". On pourra chercher autant qu'on voudra: on ne trouvera pas un seul endroit dans les livres de Latouche où il fait l'apologie de la foi. L'amalgame entre Goldsmith et Latouche est manifeste ; or les positions de ces deux auteurs sont fort différentes: Goldsmith tend à idéaliser les petites communautés autochtones et le passé, Latouche cherche une issue dans l'inventivité (et non les traditions) des peuples qui échappent encore un peu à ce qu'il appelle "l'occidentalisation du monde ». Chez Latouche on trouvera d'innombrables dénonciations de la foi dans le progrès et la croissance - et c'est bien le terme " foi » qui est utilisé par Latouche, notamment. S'il y avait lieu de discuter du concept de "croyance " chez Latouche, il aurait fallu en tenir compte. Pourquoi Jean-Marie Harribey ne s'intéresse-t-il pas aux croyances entretenues par le discours sur le progrès, notamment sur la capacité de la technique à résoudre les problèmes écologique ? N'estce pas contradictoire avec sa défense de la "durabilité forte », qui se définit justement par son pessimisme envers les capacités de "la technique" à remplacer les biens naturels, là où les partisans de la " durabilité faible » affirment au contraire que la technique résoudra tout à temps et que nous n'avons pas à modifier notre mode de vie ?

Pour appuyer sa thèse d'une volonté latouchienne de "substituer une foi à une autre ", JeanMarie Harribey évoque la conclusion du Pari de la décroissance. Là encore, le lecteur trouvera quelque chose de fort différent de ce qui est présenté par l'économiste marxiste. Si Latouche évoque la question de la croyance, c'est d'abord en relation avec la thèse de J.-P. Dupuy (Pour un catastrophisme éclairé) qui dit qu'un des problèmes aujourd'hui est que nous ne croyons pas en ce que nous savons. Le terme "croire " ici est évidemment pris dans un sens très différent de la croyance religieuse; et si on voulait analyser ce sujet dans le détail c'est vers les ouvrages de Dupuy, et non Latouche, qu'il faudrait se tourner. Dupuy a d'ailleurs écrit des livres sur la question 
du sacré, en relation notamment avec René Girard (dont les thèses sont discutables). En tout cas, là encore, ne mélangeons pas tout ! Faire des équations comme "Goldsmith parle de réenchantement, Latouche aussi, donc Goldsmith = Latouche » n'est pas sérieux.

D'autant que Latouche est très explicite sur le sujet de la croyance entendue comme " religion ». Que I'on nous permette ici une citation un peu longue: "Si, avec le sociologue français Emile Durkheim, on définit la religion de façon laïque et très large comme l'ensemble des croyances partagées qui lient une collectivité donnée, il est peu douteux que l'économie, dans le monde contemporain, entre bien dans la case des croyances ou "religions" antérieures, voire se substitue à elles et constitue une nouvelle "catholicité" (catholicos signifiant "universel"). [...] l'avènement, avec l'émergence de la modernité, d'une foi nouvelle dans le progrès et ses corollaires (la technique, la science, la croissance) [...] permet de parler véritablement de religion de l'économie. [...] si la foi dans le progrès et dans l'économie n'est plus un choix de la conscience mais une drogue à laquelle nous sommes accoutumés et à laquelle il est impossible de renoncer volontairement [...] le "réenchantement" relatif du monde engendré par la science, le progrès et le développement est désormais bien défraichi. [...] Est-il vraiment nécessaire de faire appel aujourd'hui aux théologiens, aux ayatollahs, voire aux grandes prêtresses écoféministes des cultes néopaïens syncrétiques ou aux gourous new age qui fleurissent ici et là pour meubler le vide de l'âme de nos sociétés à la dérive ? ". Qu'on nous dise ce que cette conclusion a de commun avec Goldsmith, que la citation d'Harribey mettait à égalité ! Jean-Marie Harribey devrait donc nous expliquer comment de tels propos, dans le texte de Latouche, sont compatibles avec le résumé qu'il en fait. Et cela d'autant que Latouche défend à peu près les mêmes idées depuis 20 ans, bien avant qu'on ne parle de " décroissance "; il est donc difficile de parler de surprise à son sujet.

On peut faire une tout autre généalogie, en reprenant les thèmes proposés par le texte d'Harribey, juste pour bien voir la différence :

- des « pessimistes de l'économie » ? Oui en effet la langue enchantée de l'économie et ses multiples bienfaits (voitures, Internet etc.) endort les esprits et n'empêche pas tant de concevoir les «limites naturelles » à l'accroissement de la population qui était le souci de Malthus mais surtout le fait que les inégalités augmentent et que l'environnement se dégrade. Si l'on s'inscrit dans le paradigme de la « durabilité forte » alors en effet on est "pessimiste " à l'endroit de la capacité du progrès technique à assurer l'égalité et la protection de l'environnement sans modifier le niveau de vie des plus riches, et on évite de prendre pour argent comptant le discours néolibéral sur la technique tout-puissante. Dénonciation de la société de spectacle ! De la technoscience ! Pour des techniques conviviales, autonomes, citoyennes ! Pour une réappropriation des besoins et surtout des désirs!

- des écolos ? Oui parce que la nature est une source de richesse, elle ne doit pas être envisagée comme un simple moyen de production mais comme une res communis, guidée par une éthique environnementale etc. bref toutes sortes de motivations qui ne vont pas automatiquement dériver vers le new age. Ecole de Francfort en matière de critique des sciences et techniques - Eros contre Thanatos ! On s'étonnera du refus de principe affiché de Jean-Marie Harribey face au réenchantement du monde, dont la nature joyeuse et optimiste lui a manifestement échappé. Alors quoi ? Les passions tristes ? La dictature du prolétariat?

- l'entropie ? Oui, c'est pour ça que les renouvelables sont importantes, et que l'économie doit décroître maintenant et pas plus tard : parce que si elle continue de croître, elle finira par décroître jusqu'à zéro cette fois, justement, et jusqu'à ce qui alors serait notre mort à tous! Or nous voulons vivre, tous! Donc non seulement efficacité mais surtout sobriété, désencombrement, autonomie.

\section{En conclusion}

Nous n'entendons pas démontrer ici que la mouvance qui se présente sous l'étiquette " d'objection de croissance " a une solution à tout, évidemment, ni même qu'elle est une théorie cohérente et unifiée, dotée d'un programme politique clair. Par contre nous devons nous interroger sur ces 
incompréhensions persistantes entre Jean-Marie Harribey et les objecteurs de croissance, des incompréhensions qui penchent toujours dans le même sens :

- une compréhension de la décroissance au travers des cadres étroits des catégories économiques, fussent-elles jugées « critiques » par leurs auteurs, dans un jugement qui semble autosatisfait ;

- une imperméabilité relative à une discussion fine sur ce qui constitue "le progrès », "le développement », les sciences et techniques, et plus généralement les usages, et de manière concomitante une ignorance de la réalité sociologique des mouvements écologistes, de la « démocratie technique » etc. ;

- un déni de poids ontologique à la catégorie du symbolique, au profit des « vrais » rapports de force, qui sont économiques - et par là une erreur déjà commise par Marx et le marxisme : une absence de pensée de l'Etat, qui est pourtant lourde des erreurs du passé.

Ces trois points désignent en creux non seulement ce qui distingue aujourd'hui des économistes qui cherchent à prendre en compte l'enjeu écologique des objecteurs de croissance, ancrés dans les luttes de terrain, mais aussi et surtout les lieux d'anomie à gauche. En effet aucun parti de gauche n'a aujourd'hui de discours clair sur ces différents points. Quelle économie mettre à la place du néolibéralisme ? Pour quel progrès matériel ? Comment réduire le fossé creusé au fil du temps entre les représentants et les représentés ? Autant de questions qui sont d'une brûlante actualité et dont il y a lieu de débattre plus largement et non de les occulter. 